This item was submitted to Loughborough's Research Repository by the author.

Items in Figshare are protected by copyright, with all rights reserved, unless otherwise indicated.

\title{
Designing a talents training model for cross-border e-commerce: a mixed approach of problem-based learning with social media
}

PLEASE CITE THE PUBLISHED VERSION

https://doi.org/10.1007/s10660-019-09341-y

\section{PUBLISHER}

Springer

VERSION

AM (Accepted Manuscript)

\section{PUBLISHER STATEMENT}

This is a post-peer-review, pre-copyedit version of an article published in Electronic Commerce Research. The final authenticated version is available online at: https://doi.org/10.1007/s10660-019-09341-y.

\section{LICENCE}

CC BY-NC-ND 4.0

\section{REPOSITORY RECORD}

Cheng, Xusen, Linlin Su, and Alex Zarifis. 2019. "Designing a Talents Training Model for Cross-border Ecommerce: A Mixed Approach of Problem-based Learning with Social Media”. figshare.

https://hdl.handle.net/2134/37489. 


\title{
Designing a talents training model for cross-border e-commerce: a mixed approach of problem-based learning with social media
}

\author{
Xusen Cheng ${ }^{1} \cdot$ Linlin Su$^{1} \cdot$ Alex Zarifis $^{2}$
}

\begin{abstract}
Cross-border e-commerce has developed rapidly integrating the global economy. Research has presented some solutions for the challenges and barriers in crossborder e-commerce from the perspective of the enterprise. However, little is known about the requirements of cross-border e-commerce talents and how to train them. In this paper, we firstly conducted semi-structured interviews to acquire the requirements of cross-border e-commerce talents. Business and market knowledge, technical skills, analytical ability and business practical ability were found to be the four core requirements. Then, we integrated problem-based learning and social media to design a talents training model for cross-border e-commerce and did a program to evaluate effectiveness of the model. Finally, its effectiveness was evaluated from the four evaluation dimensions of attitude, perceived enjoyment, concentration and work intention. The talents training model was improved according to the suggestions.
\end{abstract}

Keywords Cross-border e-commerce (CBEC) · Problem-based learning (PBL) . Social media $\cdot$ Talent training model

Xusen Cheng

xusen.cheng@uibe.edu.cn

Linlin Su

aaronlinbest@163.com

Alex Zarifis

alex.zarifis@kit.edu

1 School of Information Technology and Management, University of International, Business and Economics, 10 Huixin East Street, Beijing 100020, China

2 Karlsruhe Institute of Technology, 763131 Karlsruhe, Germany 


\section{Introduction}

Two traditional ways of trading, offline cross-border trade and domestic trade, have been covered extensively in previous studies [20]. With the advancement of information technology (IT), the number of domestic e-commerce research is rapidly increasing $[40,38]$. Recently, due to the integration of the global economy, cross-border e-commerce (CBEC) has developed rapidly [26]. It's reported that the market scale of CBEC was US $\$ 308$ billion in 2014, which was about $15 \%$ of the e-commerce market [1]. In 2018, the number was increased to US $\$ 675$ billion, and it was expected to reach US \$994 billion in 2020 [1]. More and more companies and individuals are trying to explore the new way of online shopping to satisfy the demand.

CBEC, which is under the Chinese Belt and Road Initiative, has obtained a lot of support from the government for its development [24]. Because of this encouragement and the huge market, many companies in other countries want to do CBEC with Chinese companies. It's also a good chance for many Chinese CBEC companies to develop the international market. However, compared to other trade mechanisms, the mechanism of CBEC is more complex [17]. For example, doing CBEC requires information technology. Furthermore, the cross-culture interaction is an essential skill. Consequently, it appears to need special CBEC talents to start a CBEC business or accomplish the work in a company. However, the rise of demand in doing CBEC indicates that many companies suffer from lacking enough staff being proficient in CBEC [36].

With the expansion of the Internet, teaching methods and studying approaches tend to be pluralized $[31,3]$. Problem-based learning (PBL), the approach that students learn theory and knowledge through solving authentic and practical problems, has been regarded as a useful teaching method [6, 25]. Meanwhile, more and more students are used to leaning with the help of several electronic tools such as computers and mobile phones. The social media is widely used by students and has a positive effect on the satisfaction of students when applied in teaching [31]. The main purpose of higher education is to cultivate talents for society [9]. Because of the current situation with insufficient talents being skilled in CBEC, many universities have set up courses about CBEC. However, most of these courses only introduce theory of CBEC, and there is not enough interaction between students and professors. It is evident that CBEC encompasses many practical contents, which means only learning theory is not enough. PBL always provides a problem scenario where students learn knowledge when they collaborate to solve problems. Therefore, it is urgent to design an appropriate training model based on PBL with social media which can be used in CBEC education in line with the requirements.

Although CBEC has been investigated by considerable research, most of them have concentrated on the consumer, the supplier and the enterprise [18, 26, 13]. It is indeed critical to analyze the mechanism of CBEC. However, it can't be neglected that the talents shortage in CBEC is an urgent issue, and there is a lack of research covering what the requirements of CBEC talents are and how to train 
this kind of talents. This paper is interested in CBEC talents, which is different from prior studies that have mainly concentrated on the trading process of CBEC. To fill this research gap, in this study, we hold the exploratory viewpoint to investigate the CBEC talents and integrate problem-based learning (PBL) and social media to design a talents training model for CBEC.

After explainin the research motivation, we address these three research questions in this paper:

RQ1: What are the requirements of CBEC talents?

RQ2: How can a talents training model for CBEC be designed?

RQ3: What is the effectiveness of the CBEC talents training model implementation?

This paper contributes to prior literature by discovering the requirements of CBEC talents and designing the training model based on the requirements and the PBL theory, which provides a new theoretic perspective on CBEC talents. In addition, we creatively combined PBL with WeChat, which is a kind of popular social media in China, when we designed the model. By successfully applying the model in teaching, this paper provides a new direction to cultivate CBEC talents which extends the knowledge of CBEC talents education.

The following sections of this paper are arranged as follows. In the next section, we review the literature in the field of CBEC and education. Then, the methodology of this paper is introduced in detail. Next, we propose the process of data collection and analysis. Subsequently, the main results of this research are discussed. Finally, we conclude with the results, their implications, the limitations and future research.

\section{Theoretical foundation}

\subsection{Cross-border e-commerce (CBEC)}

Trading by electronic means with another country rather than domestically is the typical feature of CBEC, and it is also the main difference between CBEC and traditional e-commerce [26]. Before the appearance of CBEC, it was difficult for consumers to purchase commodities from other countries. Meanwhile, it is difficult for some small and medium enterprises to be involved in international trade [37]. Consequently, the primary value of CBEC is reflected in two aspects. First, CBEC is considered to provide consumers with a facility. Second, it lowers the barrier for enterprises to do international trade. In recent years, it has been reported that the advancement of CBEC is continually on the rise [14].

The existing studies of CBEC mainly have three streams. First, the same as the other kinds of trading, the most essential point in CBEC is logistics which undertakes the responsibility of delivering an intact commodity from the supplier to the consumer and can totally influence the quality of the trade [16]. The prior literature suggests that the long distances are always the challenge of logistics in CBEC [17]. The arguments state that the long distances cause a series of issues such as 
barriers of communication, challenges to knowing the markets of the other countries and delivery costs $[17,22,26]$. It is the responsibility of those participating in a CBEC deal to overcome the problems caused by the complex logistics. In summary, the staff who are engaged in $\mathrm{CBEC}$ are required to have the ability to communicate effectively and have a keen sense and analysis of the market. However, despite most prior studies identifying barriers existing in the business of CBEC, most of them do not discuss what kind of people are needed in this context. Second, the external market environment of the CBEC is also a point that influences trade. Most existing research explores the effect of the external market environment including culture, institutional risk and early-mover advantages [14, 17, 34]. Small and medium enterprises naturally face difficulties when they encounter large enterprises with earlymover advantages [41]. However, based on earlier literature, there are several ways for small and medium enterprises to mitigate the lead large enterprises gain from early-mover advantages [14]. Consequently, when we consider the skills of staff in a CBEC enterprise, the ability to analyze the process of dealing with the early-mover advantages is stressed. Unlike domestic e-commerce, CBEC frequently involves more than one country. Furthermore, the two sides tend to have a different culture, which can cause the problem that suppliers and customers can't fully understand each other. This can make it difficult for the customers to complete the trade successfully [34]. In addition, previous studies indicate that institutional risk widely exists in CBEC [17, 37]. Therefore, it is necessary for enterprises to understand and abide by the institutions in different countries when they trade in CBEC. In line with all these challenges, the analytical ability, cross-culture communication skill and ample market knowledge must be embedded in a CBEC talent. However, to the best of our knowledge, there is not enough research focusing on all these skills which staff need for CBEC. Lastly, some prior research focuses on the intention and behavior of the supplier and customer in the context of cross-border e-commerce [18]. This kind of important viewpoint also exists in several other fields and is usually the main point focused on by researchers $[8,10,38]$.

Table 1 shows the summary of literature in CBEC. According to our literature review of the field of CBEC, most of the barriers in the process of business in CBEC are mentioned. However, the literature has contributed towards solving these problems from the perspective of the enterprise. This leaves a gap we would like to fill on how to solve these problems from the perspective of talent. This research attempts to fill this gap.

\subsection{Problem-based learning}

Problem-based learning (PBL) initially appeared in the field of medicine. It has been used in other fields such as education, management and marketing in recent years [21]. PBL is a teaching method that typically includes these steps: firstly, the professor presents an authentic problem to students who have been divided into small groups. The problem is always based on the objective of the course. Then, the students in each group discuss the problem and try their best to gather information to solve it. Finally, after the group discussion and arriving at a consensus, they present 


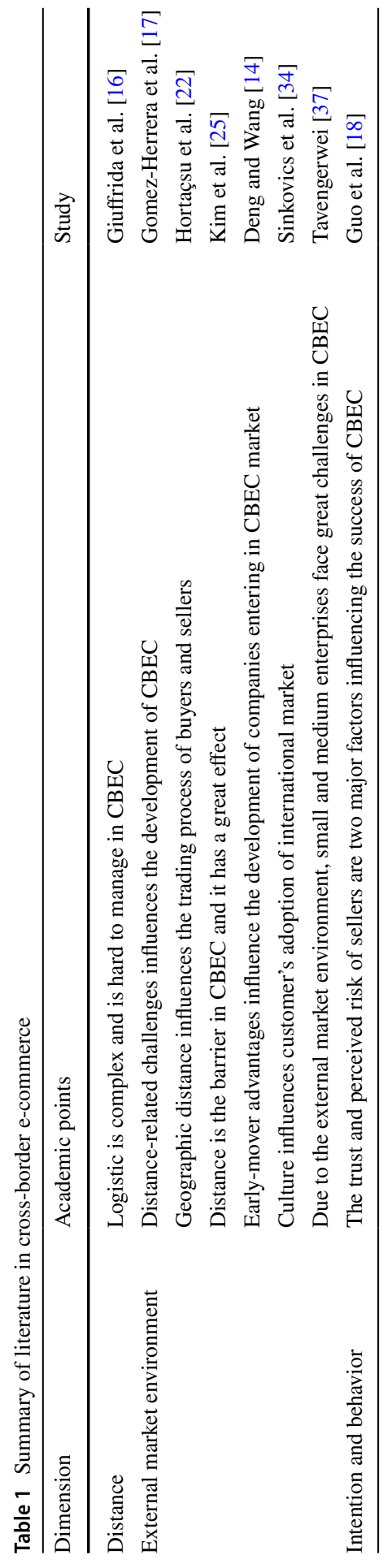


the result and obtain the feedback from the professor [32]. Consequently, PBL is the approach that helps students to deeply understand the knowledge through solving authentic problems [25]. In the process of PBL, students mainly learn through solving problems by self-directed study, and it is suggested by prior studies that the effectiveness of PBL is better than more passive, traditional teaching methods [6, 28, 42].

The core points of PBL are collaboration, self-study and flexible directing [45]. To solve the presented problem, students have enough time to collaborate with group members and to study by themselves with more flexible guidance from a professor [33]. These features of PBL make students more engaged in the problem and they learn more practical skills, which are the priority of PBL [35]. Based on what has been mentioned, practical skills are important for staff doing business in CBEC. Consequently, PBL is the suitable method for training CBEC talents. However, there is little research combining PBL and training CBEC talents together to test the effectiveness of this method. To fill this gap, this study designs a teaching method based on PBL and applies the method to teaching, to test the effectiveness of the method.

Apart from the traditional PBL teaching method, with the advancement of technology, PBL is expected to integrate online measures [30]. Several literatures have noted that computer-based scaffolding has a positive effect on the motivation and interest of students $[5,25]$. Computer-based scaffolding is a kind of guidance which can guide the student in solving a problem when the student can't get access to a professor. In addition, with the emergence of more and more new technology that can help the student in learning, prior studies have examined the positive association of technology and learning effectiveness [19, 23]. Meanwhile, in recent years, students use the mobile phone more frequently than using computer, yet little is known about how to involve these new forms of technology in PBL. This study is interested in applying a form of social media called WeChat in the process of PBL and checking the effectiveness of this combination.

\subsection{Flow theory}

The flow state is a kind of affective state that exists when a person is totally immersed in what they do [11]. When a person is in the state of flow, they can understand what they are focusing on effectively. Flow theory has been applied in many fields especially in the information technology and education field. In the field of information technology, researchers have mentioned that if people feel technology is fun and they enjoy it, they will perceive that the time flies [2]. In learning, flow state is an ideal state because people can access their maximum efficiency [12]. Consequently, this paper evaluates the effectiveness based on flow theory.

Prior research has suggested that flow is a construct with several dimensions [29]. It is widely accepted that flow is divided into three dimensions including perceived enjoyment, perceived control and concentration [27]. Perceived enjoyment and concentration reflect one's interests. Considering perceived control is more suitable for information technology rather than education, this paper uses perceived enjoyment and concentration to evaluate the effect of teaching. 


\section{Study 1: Identifying requirements of CBEC talents}

Since there is limited research in the requirements of CBEC talents, in study 1, we started our investigation by exploring the first research question [15]. Although we have reviewed existing literature and know about barriers or challenges in CBEC, we need more evidence to identify the requirements of CBEC talents. In this study, we elicited the answer to the first question by means of conducting semi-structure interviews and analyzing the data.

\subsection{Study 1: Method}

We conducted interviews in study 1. Firstly, we designed interview protocols according to our research questions. Then, we did the semi-structured interviews and analyzed the data. Finally, we discussed and made a consensus about the requirements of CBEC talents.

\subsection{Study 1: Data collection}

We collected data mainly from three sources, documents, interviews and previous literature. The three sources of data were used to triangulate and to improve validity [44]. The documents include courses in the area of information management and e-commerce all over the world and information in CBEC companies. By reading through these documents, we improved our understanding of existing courses and the current situation of CBEC companies. After the semi-structured interviews, we also integrated these two sources of insight to support our analysis.

Four managers of four different CBEC companies and two experienced academic professors were interviewed. All of them were invited to participate in a project of a giant e-commerce company so that we had the chance to interview them. Table 2 shows the basic information of those interviewees. Each interview was recorded and transcribed for analysis. The interview protocols for managers and academic professors were a little different, but both mainly included three parts. In the protocols of managers, first, we asked information about their companies. Then, we inquired about the barriers or challenges when they do CBEC. We also learned about their approach in dealing with these problems. Lastly, we asked about skills which staff working in their companies needed and suggestions about how to acquire these

Table 2 Interviewees list

\begin{tabular}{lll}
\hline Number & Country & Job role \\
\hline M1 & Thailand & Manager \\
M2 & Thailand & Manager \\
M3 & Korea & Manager \\
M4 & Indonesia & Manager \\
A1 & Indonesia & Academic Professor \\
A2 & Malaysia & Academic Professor \\
\hline
\end{tabular}


skills. In the interviews of academic professors, first, we asked about information about academic professors in their university. After that, we inquired about information about students learning in e-commerce. Finally, we asked them about the teaching content. We will use a specific number to stand for each interviewee for convenience. For example, the first interviewee will be marked as M1 which means the abbreviation of Manager 1 and A1 means the abbreviation of Academic Professor 1.

\subsection{Study 1: Data analysis}

Utilizing the documents we mentioned before and the process of analyzing the interview data, we uncovered the requirements of CBEC talents. We analyzed the interview data in terms of three steps. Table 3 shows the examples of how we analyzed all the interview data.

Step 1: Synthesizing Since the interview data was dispersive and consisted of some irrelevant information, the authors of this paper firstly synthesized all the interview data and reviewed it carefully to identify information related to the requirements of CBEC talents. Two of the authors and the other seven people in the project participated in this work to make sure the results were accurate.

Step 2: Conceptualizing and classifying Many of the sentences in the interview data were long and colloquial. For the demands of this investigation, they needed to be conceptualized into short phrases and classified according to the meaning. One of the authors conceptualized and classified the results of the first step, and the other authors helped to cross-check this analysis. After that, they discussed with the first author until they arrived at a consensus.

Step 3: Extracting After the two steps of the data analysis, we put together the core points of the data. Finally, we extracted the requirements of CBEC talents from the results of the step 2 linking them with the literature.

\subsection{Study 1: Results}

The qualitative analysis identified four components of core requirements of CBEC talents. Figure 1 presents the four components. This section firstly explains these four aspects and then introduces some suggestions acquired from the interview data.

\subsubsection{Business and marketing knowledge}

Business and marketing knowledge are hard to define since they are comprised of a wide range of knowledge. Business and marketing literature have covered many aspects of business and are vast concepts [43].

In CBEC, business and marketing knowledge are referred to in many aspects by the interviewees. For example, the knowledge of the environment of trading countries and academic knowledge. Two related statements made by interviewees were:

I am investigating and learning the relevant market knowledge. I am finding whether I can enter in another new market, and I spent more than 6 months to 


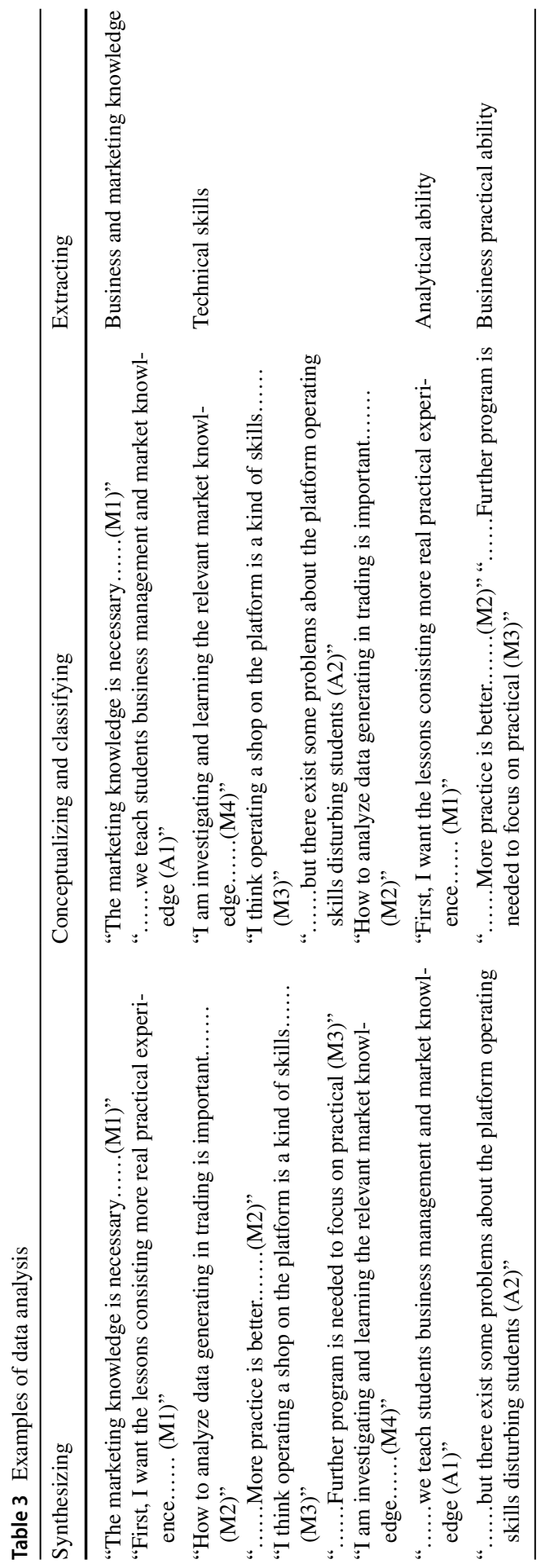




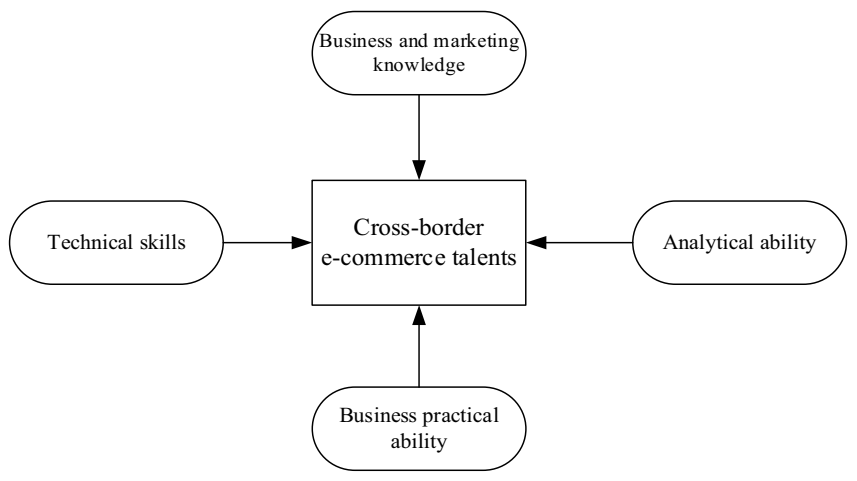

Fig. 1 Core requirements of CBEC talents

learn about the Indian market. In the future, maybe I will expand into Chinese market (M4).

In the business and academic program, we teach students business management and market knowledge (A1).

Meanwhile, the knowledge of managing the client relationship and emerging Internet marketing were also mentioned:

The marketing knowledge is necessary, and they also need to know how to manage client relationship. It is important for our company (M1).

I am not familiar with Internet marketing and IT technology, but it is better to learn them (M4).

\subsubsection{Technical skills}

With the rapid development of the Internet, relevant technical skills have gained attention. For an organization to be successful in CBEC, it is evident that technical skills must be paid more attention.

Since CBEC takes the online platform as an intermediary, it requires significant technical skills for trading. Some interviewees mentioned:

I think all of the technical (staff needs to learn) should be classified, and one of them is operating the online shop (M4).

In the project, we teach students operational skills in the second stage. The objective is to cultivate them to be the e-commerce ecosystem builder or creators, but there exist some problems about the platform operating skills disturbing students (A2).

To know payment method is important to staff...... The logistics is a problem, and logistics is important for cross-border trading (M2).

I think operating a shop on the platform is a kind of skills, and it's not a kind of difficulty to me. (M3) 


\subsubsection{Analytical ability}

Analytical ability is considered necessary to figuring out the solution to difficult problems in many disciplines. Faced with a complex world, analytical ability is critical to making the right decisions.

For the complex situation in CBEC, it appears that problems existing in CBEC commonly consist of several different components. Dealing with these kind of problems is not easy. It requires that the decision-maker has good analytical skills. Some interviewees said:

How to analyze data generating in trading is important. The owner of enterprise needs data analytical skills (M2).

We need plenty of case studies to help students learn more about the CBEC (A2).

The E6 interviewee also mentioned that teaching students with case studies was more effective, since the case studies derived from real e-commerce trading closely, so students would be more interested in them.

\subsubsection{Business practical ability}

Business practical ability is the overriding capability for a professional in business and directly influences the effectiveness of trading. In CBEC, business practical ability refers to the ability to implement the processes of a CBEC enterprise. The interviewees emphasized the business practical ability, considering that the most important skill is the practical skill. Some of them said:

First, I want the lessons consisting more real practical experience. Second, after getting knowledge, I will do things to practice myself (M1). (about the teaching method) More practice is better. Other than teaching knowledge, practicing is good (M2).

The courses were too much focusing on strategy. Further program is needed to focus on practical (M3).

Consequently, only learning theory in a class is not enough for staff occupied in CBEC.

\subsubsection{Teaching environment}

Based on what was mentioned earlier, combining all this knowledge with practical practice is the best way to train CBEC talents. Additionally, we acquired some suggestions about the environment of the course setting. Some interviewees reported:

Compared to large scale teaching, studying in small group is easier to understand and more effectiveness......In the program, many countries and many cultures get together and interact in an open office. I think it is good (M1). 
I prefer choosing the way of group discussion. In this way, we can get more interactions and more practice (M2).

Based on the analysis of the results we integrated the core capabilities and the teaching environment in CBEC together as illustrated in Fig. 2.

\section{Study 2: Designing the training model for CEBC talents}

In study 1, we identified the four core requirements of CBEC talents and suggestions about the teaching environment. Combining the results of study 1 and our previous discussion of PBL $[4,5]$, we designed the training model for CEBC talents. In addition, social media applications have been focused on and applied in teaching [7, 31]. Consequently, we applied the social media application in the process of teaching. This section is divided into three components. Firstly, we introduce the method we used to design the course for training CBEC by applying social media. After that, we describe the experiment in detail. Furthermore, we identify the method of evaluating the effectiveness of the course.

\subsection{Study 2: Method}

PBL has been demonstrated as a teaching framework consisting of a problem, knowledge, related information and a solution, which is appropriate for enhancing technical skills and analytical ability [5]. Social media is widely used by students and has a positive effect on the satisfaction of students when applied in teaching [31]. Consequently, we designed the training model for CEBC talents by applying social media application according to the results of study 1 and the theory of PBL. Apart from these view points, to enhance the business practical ability, we added the part of writing a business plan in the training model.

An experiment was conducted to answer the last two research questions. The objective of the training model is to train CBEC talents and stimulate them to do

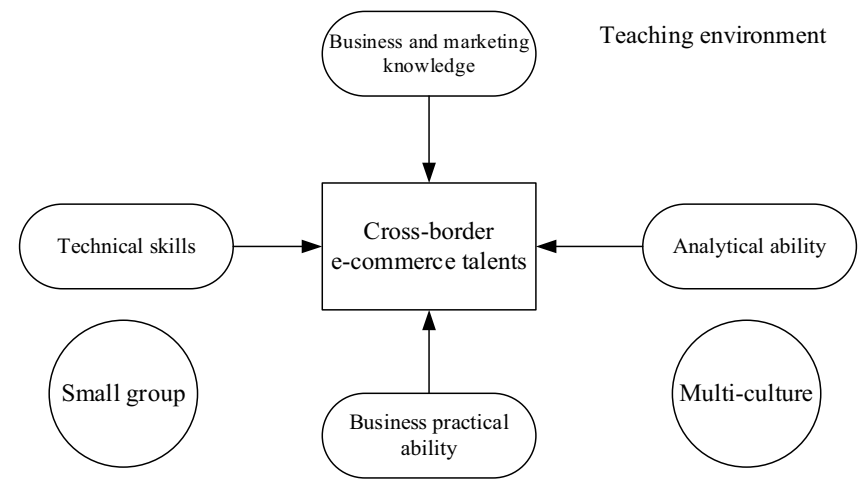

Fig. 2 Core requirements and teaching environment 
CBEC business. Since we designed the training model for CBEC talents, it was necessary for us to do an experiment and test the effectiveness of the course.

We applied the model in a program to train students in a Chinese university. Graduate students from different countries participated in our experiment. Although they were from different countries and share different cultures, they had similar characteristics such as competence and age. They could all communicate in English. They attended the program and were taught by the training model we provided. Firstly, they were divided into two groups randomly for group collaboration. Meanwhile, they had enough time to get familiar with their groupmates. Then, the professor gave them a case study about a platform of CBEC and asked the students to find problems existing in the platform and think about the resolution of those problems. They were also given the task of writing a business plan about doing CBEC. After that, they had two classes for each week. In class, the professor taught them about business and market knowledge related to CBEC. In addition, they could ask the professor any questions and talked about all the difficulties they met in discussing the problem. Finally, the students could discuss face-to-face with their groupmates before and after the class. In addition, the professor and students were in a WeChat group so that students could easily ask the professor for help after class. They also discussed with their groupmates and transferred files by WeChat. Two months later, they were asked to present the problems they found in the case. After 4 months, they presented the solution for those problems and submitted the business plan. At the end of the experiment, the professor gave feedback to the students. Students got the course grade with comments from the professor. To evaluate the effectiveness of the course, we conducted interviews with these students.

\subsection{Study 2: CBEC training model}

We present the process of the CBEC training model in Fig. 3. Following the illustration, the problem related to the objective of the program is presented first. Then, students try to find the solution to the problem by gaining knowledge and group collaboration. In the group collaboration, they can have a face-to-face discussion and online discussion using WeChat. They find related information through various channels. The group collaboration is an iterative process. Knowledge is gained by students during the time they try to solve the problem, including knowledge from the professor, classmates, friends and the Internet. Students collaborated to solve the problem and wrote the business plan about CBEC at the same time.

The training model that was designed based on PBL with social media enhances the three components of ability we mentioned in the last section. Meanwhile, writing a business plan enhances all four components of ability.

\subsection{Study 2: Data collection}

Interviews were conducted to evaluate the effectiveness of the training model. Nine students in nine different countries were interviewed. Table 4 shows some 


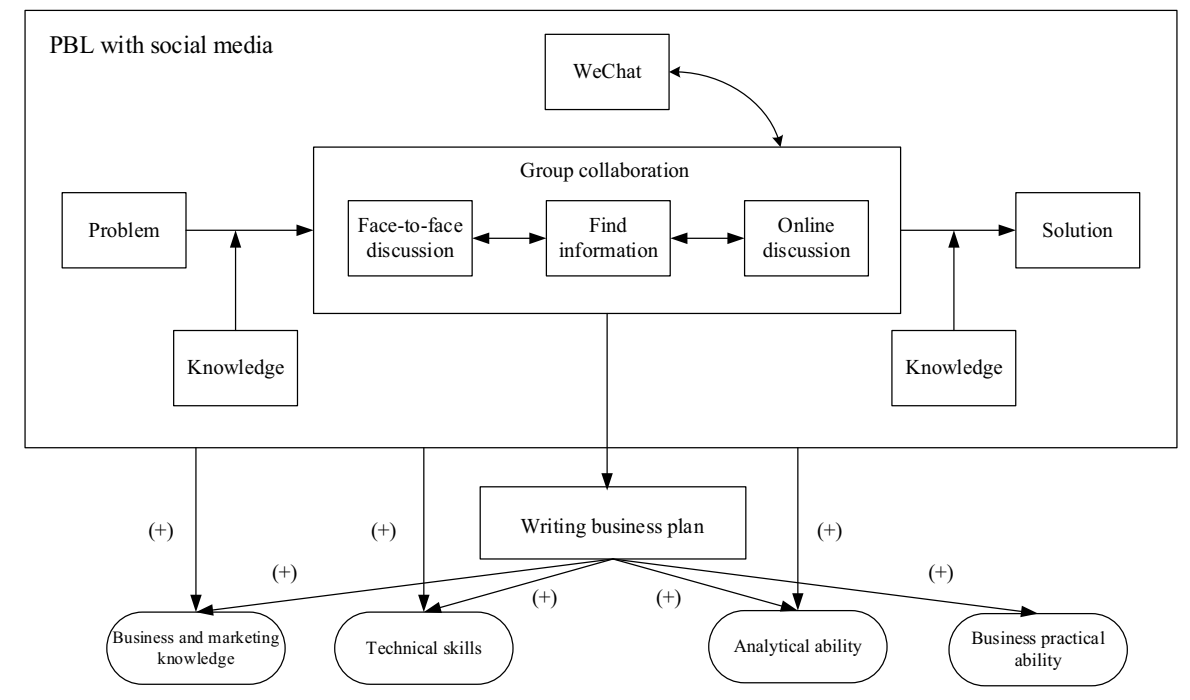

Fig. 3 CBEC training model

Table 4 Interviewees list

\begin{tabular}{lll}
\hline Number & Nation & Gender \\
\hline S1 & Russia & Male \\
S2 & Azerbaijan & Female \\
S3 & Indonesia & Male \\
S4 & Pakistan & Female \\
S5 & Kazakhstan & Female \\
S6 & Thailand & Female \\
S7 & Jamaica & Female \\
S8 & Rwanda & Female \\
S9 & Mongolia & Female \\
\hline
\end{tabular}

basic information of those interviewees. Each interview was recorded and transcribed for analysis. In line with flow theory, perceived enjoyment and concentration reflect one's study state. Based on flow theory and the objective of the program, the interview protocol mainly comprised of four components. Firstly, we inquired about their attitude to the program. Then, we asked about the process of discussing and solving the problem. In the third part, we asked about their intention to work in the field of CBEC. Finally, we inquired about their suggestions on the training model. The former three parts were intended to get information about their attitude. The last one was intended to acquire suggestions. We will use ' $\mathrm{S}$ ' plus a number to stand for each interviewee for convenience. For example, the first interviewee will be marked as S1. 


\subsection{Study 2: Data analysis}

The purpose of the data analysis is firstly to evaluate the effectiveness of the training model. The second purpose is to improve the training model according to the suggestions from the students. We analyzed the interview data in terms of three steps, as described below.

Step 1: Classifying The initial interview data included some irrelevant sentences and was undisciplined. One of the authors classified the sentences which were related to the research questions into two components of attitude and suggestions. The other authors helped to check the results and they discussed them until they reached an agreement.

Step 2: Extracting After step 1, we had classified sentences. Then, we refined the key points from these classified sentences. One of the authors extracted the keywords and the other authors helped to check, which was similar with step 1.

Step 3: Drawing conclusion Combining the key points, we finally drew the conclusion about the effectiveness of the training model and improved it.

\subsection{Study 2: Result}

This section presents the result of the data analysis and mainly has two aspects: firstly, the attitude of students towards the training model was used to evaluate its effectiveness. Secondly, the suggestions were used to improve the training model.

\subsubsection{Effectiveness of the training model}

The attitudes towards the training model were positive. They all expresses that this kind of teaching method was different from the others, and they really acquired extensive new knowledge about CBEC from the class.

The answers on the process covered how they discussed and solved the problem and were both positive. Based on these answers Table 3 shows the state when they were trying to solve the problem and were writing the business plan. We express them from two aspects as perceived enjoyment and concentration according to flow theory.

What surprised us was that they all had the intention to get a job or set up their own enterprises in the field of CBEC or e-commerce after they completed the course.

\subsubsection{Suggestions}

To improve our training model, we asked the students questions to elicit their suggestions. Many of the students mentioned that they needed feedback and guidance timely. Some of them reflected that it would be better to have experience in real CBEC enterprises. 


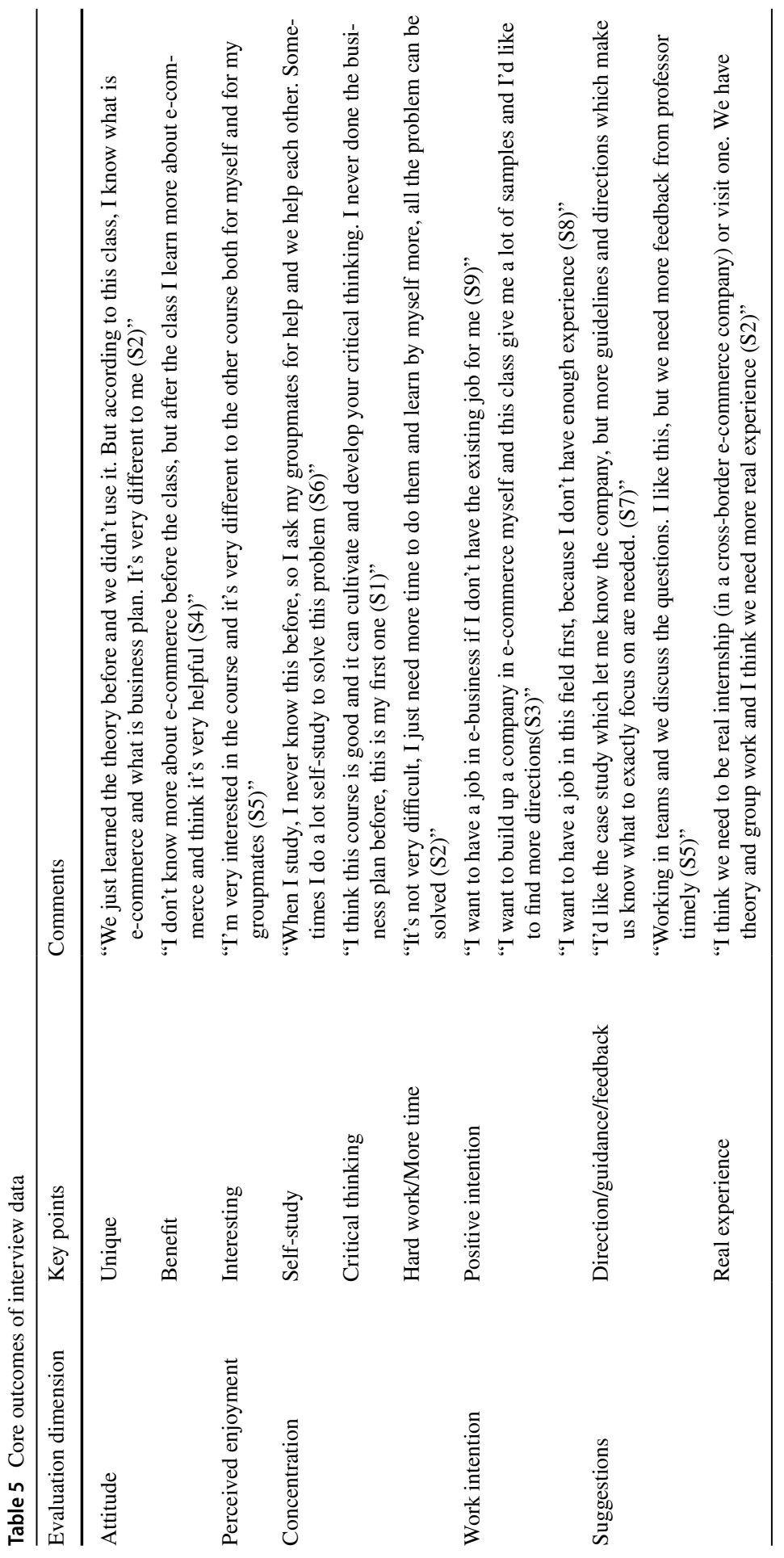


The key points of their attitude and suggestions are illustrated in Table 5 and they are also the main findings of this part. The results suggest that the effectiveness of the course is positive and is helpful to students working in the field of CBEC. Furthermore, according to those suggestions, we improved our training model and proposed the updated model in Fig. 4.

\section{Discussion}

\subsection{Key findings}

This research was motivated by the rapid development of CBEC and the lack of talents in this field.

The overall study intends to expand the knowledge about CBEC talents. We completed two studies to explore and answer the three research questions we mentioned before. According to the results of these two studies, we express our findings by answering the three questions.

RQ1: What are the requirements of CBEC talents?

Talents play a pivotal role in CBEC business. Many studies have demonstrated the importance of skills in doing CBEC business. However, to the best of our knowledge, it's complex to express the requirements of CBEC talents. We found four core the requirements of CBEC talents by conducting semi-structured interviews. Firstly, having business and marketing knowledge is critical to CBEC talents. Both theoretical and practical knowledge are important components in business and marketing

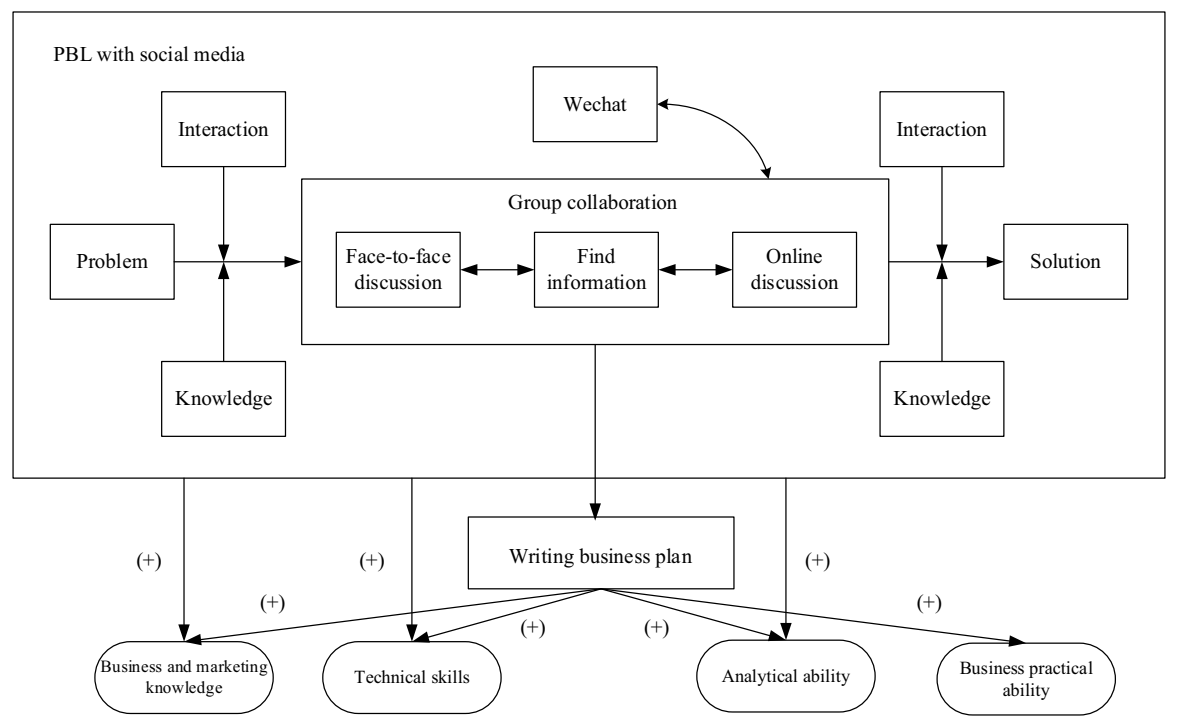

Fig. 4 Improved CBEC training model 
knowledge. Secondly, technical skills for trading online was a necessary skill for CBEC talents. Dealing with all the problems in the process of online trading proficiently is another critical skill to CBEC talents, which is comprised in technical skills. Thirdly, it's common to handle difficult situations when doing CBEC business, hence analytical ability is needed for them to solve the problem or make decisions. Last but not least, all these skills we mentioned are not independent of business practical ability which is the core the requirement. In summary, business and marketing knowledge, technical skills, analytical ability and business practical ability were found to be the four core requirements of CBEC talents.

\section{RQ2: How can a talents training model for CBEC be designed?}

After finding the four core requirements, we analyzed several teaching methods, then we chose PBL which commonly includes knowledge, real problems and learning based on solving real problems. Business and marketing knowledge, technical skills, analytical ability and business practical ability were found to be the four core requirements of CBEC talents as we mentioned before. Consequently, PBL was the most suitable theory framework for designing the training model. Meanwhile, we applied WeChat in the training model. Writing a business plan was also applied in the training model as the way of practicing practical skills. The training model is problem oriented accompanied with learning knowledge and discussing. The objective of the training model is to train the four core requirements of students and stimulate them to do CBEC business. We did a program using the model to train students in a Chinese university (Table 4).

RQ3: What is the effectiveness of the CBEC talents training model implementation?

The effectiveness of the model was evaluated from the four evaluation dimensions of attitude, perceived enjoyment, concentration and work intention. The results of the evaluation amplify the three aspects of the conclusion. First, students have a positive attitude towards the training model. Second, students are involved in the process of solving the problem and writing a business plan. Third, students have the intention to get a job or set up their own enterprise in the field of CBEC or e-commerce after they participated in the training model (Table 5).

\subsection{Theoretical implications}

While many problems have existed in CBEC such as barriers to communication, challenges to knowing the markets of the other countries and delivery costs caused by long distance and problems from the other dimensions such as external market environment including culture, institutional risk and early-mover advantages, the extant literature has focused on the barriers only and has analyzed the problems of the external market environment from the perspective of consumers or sellers. Drawing on the theory of PBL, this paper provides a new perspective of talents training 
to solve the problems we mentioned before. This research contributes to existing literature from two aspects.

First, previous studies provided barriers caused by the long distance in CBEC. However, most of them didn't present the clear resolutions [17, 22, 26]. Meanwhile, some literature contributed to solve problems in CBEC from the perspective of enterprise [14, 41]. Our research investigates how to solve these problems from the perspective of talent training. We found business and market knowledge, technical skills, analytical ability and business practical ability to be the four core requirements of CBEC talents. Identifying these four requirements contributes to the theory of CBEC from the perspective of CBEC talents and deepens our knowledge on how to deal with the challenges and remove the barriers of CBCE.

It is suggested by prior studies that the effectiveness of PBL is excellent, and most of them used the computer as scaffold [6, 42]. However, PBL is usually used in the field of education as a single method and was never used in the field of CBEC talents training. The second theoretic contribution of this paper is that we present a training model based on the theory of PBL. Our training model combined PBL with practical business exercises, which enriched the theory of PBL. Furthermore, our investigation combined social media with PBL and successfully extended this kind of method to the field of CBEC talents training, which develops a new scaffold of PBL and deepens our knowledge in PBL.

Prior research has suggested that flow is a construct with several dimensions and has divided flow into three dimensions [27, 29], but there is very limited research applying flow theory to evaluate the effectiveness of education. This paper creatively applied flow theory to the evaluation, which enriches the application of the theory.

\subsection{Practical implications}

This study provides four important implications for practice. Firstly, our results identify business and market knowledge, technical skills, analytical ability and business practical ability as core requirements of CBEC talents. Following this result, entrepreneurs can utilize the four important dimensions of CBEC talents to select a candidate applying for a job in the field of CBEC. Furthermore, they can train their staff to work better in the field of CBEC.

Universities have the responsibility of training the necessary talents for society, however, there exist a lack of talents as we mentioned before. The second significant practical implication is that professors can use the four core components to design a training model for teaching students and tasks for examining them. Furthermore, we tested our proposed training model and found it to be effective. It also motivated students to do CBEC business. To cultivate more talents for society, a professor can adopt this training model.

A third key implication is to establish a relevant professional certification and standards. Nowadays, there are no standards to evaluate the ability of people working in the field of CBEC. When training talents in CBEC, some standards are needed to evaluate the results of the training and provide them with a professional 
certification to certify their ability. Our results propose the four core abilities which can be used to establish a relevant professional certification and standards.

Lastly, according to the results of the evaluation, the training model is not only an effective new teaching method which can be reused and adapted by professors, but it is also a new way to elevate the self-oriented motivation and interest of students. This motivation encourages students to gain more knowledge both during and after the module. In the age of artificial intelligence, the results can be useful to develop the software of education, which can help more people to have a chance to learn by themselves.

\subsection{Limitation and future research}

Several limitations exist in this study. First, we didn't control the gender of the students in our experiment because the conditions did not allow this. This could influence the results of the collaboration, and furthermore, influence the results of experiment. However, due to the limitation of the conditions, we couldn't do this kind of experiment in larger ranges of students. Second, all the data analysis was based on interview data. Although several researchers analyzed the data and came to a consensus, some cognition bias could exist. However, we tried to compare different forms of data such as documents and literature to reduce this kind of bias.

In the future, we will implement this training model to larger ranges of students with different cultures to test the effectiveness and further improve the training model. In addition, it will be interesting to conduct a quantitative study to investigate the psychology and behavior in the process of training. Lastly, exploring how to improve the process of collaboration is an interesting direction for future research.

Acknowledgements We thank the National Natural Science Foundation of China (Grant No. 71571045), the Fundamental Research Funds for the Central Universities in UIBE (Grant No. CXTD10-06), Program for Excellent Talents in UIBE (Grant No. 18JQ04), and the Foundation for Disciplinary Development of SITM in UIBE for providing funding for part of this research.

\section{References}

1. Accenture and AliResearch. (2016). Global Cross Border B2C e-Commerce Market 2020 : Report highlights \& methodology sharing. Retrieved January 1, 2019 from http://unctad.org/meetings/en/ Presentation/dtl_eweek2016_AlibabaResearch_en.pdf. Accessed 22 March 2018.

2. Agarwal, R., \& Karahanna, E. (2000). Time flies when you're having fun: Cognitive absorption and beliefs about information technology usage. MIS Quarterly, 24(4), 665-694.

3. Bandera, C. (2017). Value-added service providers for mobile education: Empirical challenges and analytics. Electronic Commerce Research, 17(2), 317-333.

4. Belland, B. R., Glazewski, K. D., \& Richardson, J. C. (2008). A scaffolding framework to support the construction of evidence-based arguments among middle school students. Educational Technology Research and Development, 56(4), 401-422.

5. Belland, B. R., Kim, C., \& Hannafin, M. J. (2013). A framework for designing scaffolds that improve motivation and cognition. Educational Psychologist, 48(4), 243-270.

6. Capon, N., \& Kuhn, D. (2004). What's so good about problem-based learning? Cognition and Instruction, 22(1), 61-79. 
7. Cheng, X., Fu, S., \& de Vreede, G. J. (2017). Understanding trust influencing factors in social media communication: A qualitative study. International Journal of Information Management, 37(2), $25-35$.

8. Cheng, X., Fu, S., \& Druckenmiller, D. (2017). Trust development in globally distributed collaboration: A case of US and Chinese mixed teams. Journal of Management Information Systems, 33(4), 978-1007.

9. Cheng, X., Fu, S., Han, Y., \& Zarifis, A. (2017). Investigating the individual trust and school performance in semi-virtual collaboration groups. Information Technology \& People, 30(3), 691-707.

10. Cheng, X., Fu, S., Sun, J., Bilgihan, A., \& Okumus, F. (2019). An investigation on online reviews in sharing economy driven hospitality platforms: A viewpoint of trust. Tourism Management, 71, 366-377.

11. Csikszentmihalyi, M. (1977). Beyond boredom and anxiety. San Fransisco: JosseyBass.

12. Csikszentmihalyi, M. (2013). Flow: The Psychology of happiness. London, UK: Random House.

13. Cui, Y., Mou, J., Cohen, J., \& Liu, Y. (2019). Understanding information system success model and valence framework in sellers' acceptance of cross-border e-commerce: a sequential multi-method approach. Electronic Commerce Research. https://doi.org/10.1007/s10660-019-09331-0.

14. Deng, Z., \& Wang, Z. (2016). Early-mover advantages at cross-border business-to-business e-commerce portals. Journal of Business Research, 69(12), 6002-6011.

15. Eisenhardt, K. M. (1989). Building theories from case study research. Academy of Management Review, 14(4), 532-550.

16. Giuffrida, M., Mangiaracina, R., Perego, A., \& Tumino, A. (2017). Cross-border B2C e-commerce to Greater China and the role of logistics: A literature review. International Journal of Physical Distribution \& Logistics Management, 47(9), 772-795.

17. Gomez-Herrera, E., Martens, B., \& Turlea, G. (2014). The drivers and impediments for CBEC in the EU. Information Economics and Policy, 28, 83-96.

18. Guo, Y., Bao, Y., Stuart, B. J., \& Le-Nguyen, K. (2018). To sell or not to sell: Exploring sellers' trust and risk of chargeback fraud in cross-border electronic commerce. Information Systems Journal, 28(2), 359-383.

19. Gupta, S., \& Bostrom, R. (2013). Research note-An investigation of the appropriation of technology-mediated training methods incorporating enactive and collaborative learning. Information Systems Research, 24(2), 454-469.

20. Head, K., \& Mayer, T. (2014). Gravity equations: Workhorse, toolkit, and cookbook. Handbook of international economics (Vol. 4, pp. 131-195). Amsterdam: Elsevier.

21. Hmelo-Silver, C. E., Duncan, R. G., \& Chinn, C. A. (2007). Scaffolding and achievement in problem-based and inquiry learning: a response to Kirschner, Sweller, and Clark (2006). Educational Psychologist, 42(2), 99-107.

22. Hortaçsu, A., Martínez-Jerez, F., \& Douglas, J. (2009). The geography of trade in online transactions: Evidence from eBay and mercadolibre. American Economic Journal: Microeconomics, 1(1), 53-74.

23. Hu, P. J. H., \& Hui, W. (2012). Examining the role of learning engagement in technology-mediated learning and its effects on learning effectiveness and satisfaction. Decision Support Systems, 53(4), 782-792.

24. Huang, Y. (2016). Understanding China's belt \& road initiative: Motivation, framework and assessment. China Economic Review, 40, 314-321.

25. Kim, N. J., Belland, B. R., \& Walker, A. E. (2017). Effectiveness of computer-based scaffolding in the context of problem-based learning for stem education: Bayesian meta-analysis. Educational Psychology Review, 4, 1-33.

26. Kim, T. Y., Dekker, R., \& Heij, C. (2017). Cross-border electronic commerce: Distance effects and express delivery in European Union markets. International Journal of Electronic Commerce, 21(2), 184-218.

27. Koufaris, M. (2002). Applying the technology acceptance model and flow theory to online consumer behavior. Information Systems Research, 13(2), 205-223.

28. Loyens, S. M., Jones, S. H., Mikkers, J., \& Van Gog, T. (2015). Problem-based learning as a facilitator of conceptual change. Learning and Instruction, 38, 34-42.

29. Lu, Y., Zhou, T., \& Wang, B. (2009). Exploring Chinese users' acceptance of instant messaging using the theory of planned behavior, the technology acceptance model, and the flow theory. Computers in Human Behavior, 25(1), 29-39. 
30. Richardson, J. T. (2017). Student learning in higher education: A commentary. Educational Psychology Review, 29(2), 353-362.

31. Rueda, L., Benitez, J., \& Braojos, J. (2017). From traditional education technologies to student satisfaction in Management education: A theory of the role of social media applications. Information \& Management, 54(8), 1059-1071.

32. Schmidt, H. G., Loyens, S. M., Van Gog, T., \& Paas, F. (2007). Problem-based learning is compatible with human cognitive architecture: Commentary on Kirschner, Sweller, and. Educational Psychologist, 42(2), 91-97.

33. Schmidt, H. G., Van der Molen, H. T., Te Winkel, W. W., \& Wijnen, W. H. (2009). Constructivist, problem-based learning does work: A meta-analysis of curricular comparisons involving a single medical school. Educational Psychologist, 44(4), 227-249.

34. Sinkovics, R. R., Yamin, M., \& Hossinger, M. (2007). Cultural adaptation in cross border E-commerce: A study of German Companies. Journal of Electronic Commerce Research, 8(4), 221-235.

35. Svinicki, M. D. (2007). Moving beyond "it worked": The ongoing evolution of research on problembased learning in medical education. Educational Psychology Review, 19(1), 49-61.

36. Tarique, I., \& Schuler, R. S. (2010). Global talent management: Literature review, integrative framework, and suggestions for further research. Journal of World Business, 45(2), 122-133.

37. Tavengerwei, R. (2018). Using trade facilitation to assist MSMEs in E-commerce in developing countries. Journal of International Economic Law, 21(2), 349-378.

38. Tian, Y., Ye, Z., Yan, Y., \& Sun, M. (2015). A practical model to predict the repeat purchasing pattern of consumers in the c2c e-commerce. Electronic Commerce Research, 15(4), 571-583.

39. Tsai, H. T., Chien, J. L., \& Tsai, M. T. (2014). The influences of system usability and user satisfaction on continued internet banking services usage intention: Empirical evidence from taiwan. Electronic Commerce Research, 14(2), 137-169.

40. Wan, Y., Ma, B., \& Pan, Y. (2018). Opinion evolution of online consumer reviews in the e-commerce environment. Electronic Commerce Research, 18(2), 291-311.

41. Wang, S., Cavusoglu, H., \& Deng, Z. (2016). Early mover advantage in e-commerce platforms with low entry barriers: The role of customer relationship management capabilities. Information \& Management, 53(2), 197-206.

42. Wijnia, L., Loyens, S. M., Van Gog, T., Derous, E., \& Schmidt, H. G. (2014). Is there a role for direct instruction in problem-based learning? Comparing student-constructed versus integrated model answers. Learning and Instruction, 34, 22-31.

43. Wright, S., \& Calof, J. L. (2006). The quest for competitive, business and marketing intelligence: A country comparison of current practices. European Journal of Marketing, 40(5/6), 453-465.

44. Yin, R. K. (2009). Case study research: Design and methods (4th ed.). Thousand Oaks, CA: Sage Publication.

45. Zhang, M., Lundeberg, M., \& Eberhardt, J. (2011). Strategic facilitation of problem-based discussion for teacher professional development. Journal of the Learning Sciences, 20(3), 342-394. 